\title{
Left Ventricular Myocardial Deformation in Sepsis or Septic Shock: An Observational Study of Serial Changes by Three-Dimensional and Two- Dimensional Echocardiography
}

\section{Mingxing Xie ( $\nabla$ xiemx@hust.edu.cn)}

Department of Ultrasound Medicine, Union Hospital, Tongji Medical College, Huazhong University of Science and Technology

\section{Xiaojun Yan}

Department of Ultrasound Medicine, Union Hospital, Tongji Medical College, Huazhong University of Science and Technology

\section{Yuman Li}

Department of Ultrasound Medicine, Union Hospital, Tongji Medical College, Huazhong University of Science and Technology

\section{Juanjuan Liu}

Department of Ultrasound Medicine, Union Hospital, Tongji Medical College, Huazhong University of Science and Technology

\section{Ting Zhou}

Department of Critical Care Medicine, Union Hospital, Tongji Medical College, Huazhong University of Science and Technology

\section{Yi Zhou}

Department of Ultrasound Medicine, Union Hospital, Tongji Medical College, Huazhong University of Science and Technology

\section{Wei Sun}

Department of Ultrasound Medicine, Union Hospital, Tongji Medical College, Huazhong University of Science and Technology

\section{Chenchen Sun}

Department of Ultrasound Medicine, Union Hospital, Tongji Medical College, Huazhong University of Science and Technology

\section{Jing Ma}

Department of Ultrasound Medicine, Union Hospital, Tongji Medical College, Huazhong University of Science and Technology

\section{Li Zhang}

Department of Ultrasound Medicine, Union Hospital, Tongji Medical College, Huazhong University of Science and Technology 


\section{You Shang}

Department of Critical Care Medicine, Union Hospital, Tongji Medical College, Huazhong University of Science and Technology

\section{Research Article}

Keywords: Sepsis, Septic shock, Myocardial deformation, Speckle tracking echocardiography

Posted Date: February 2nd, 2022

DOI: https://doi.org/10.21203/rs.3.rs-1294828/v1

License: (c) (i) This work is licensed under a Creative Commons Attribution 4.0 International License.

Read Full License 


\section{Abstract}

\section{Background}

Left ventricular myocardial deformation develops dynamically when circulatory system is disturbed. What is less clear are the nature and clinical fetures of the deformation., and a consensus is yet to be reached for an ideal treatment plan. This study aims to explore the development of left ventricular myocardial deformation in septic patients by observing the serial changes in three- and two-dimensional strain parameters.

\section{Methods}

In this single-centered, prospective, and observational study, we eventually included 103 patients diagnosed with sepsis or septic shock at the intensive care unit (ICU) from December 2020 until December 2021. Demographic data, medicine, laboratory and ultrasound values were collected and measured on the 1st, 2nd, 3rd, 5th, 7th and 10th days after sepsis or septic shock. A total of 88 adult patients for 2D-STE and 3D-STE were divided into two groups according to their wall motion status: the non-hypokinetics group and the secondary hypokinesia group.

\section{Results}

Patients underwent both 3D and 2D echocardiography to evaluate left ventricular function. Comparison of four time points between the two groups showed that 2D-LVEF demonstrated a progressive, stepwise improvement each time point assessed $\left(P_{\text {time }}=0.033, P_{\text {group }}=0.041\right.$, respectively). Although 3D-LVEF slightly underestimated the LV volumes at each time point, it displayed a same changing tendency during the 10 days $\left(P_{\text {time }}=0.002, P_{\text {group }}=0.011\right.$, respectively $)$. Then, we used both 3D and 2D STE to observe left ventricular myocardial function and validated the applicability and feasibility of 3D-STE: 2D-LVGLS $P_{\text {time }}=0.000, P_{\text {group }}=0.018$, respectively, 2D-LVGCS $P_{\text {time }}=0.000, P_{\text {group }}=0.332$ respectively; 3D-LVGLS $P_{\text {time }}=0.000, P_{\text {group }}=0.391$, respectively, 3D-LVGCS $P_{\text {time }}=0.022, P_{\text {group }}=0.362$ respectively. The level of strain first decreased and then increased in these two groups during hospitalization, but both $3 \mathrm{D}$ and $2 \mathrm{D}$ strain imaged at the 10th day assessed were lower compared to that of normal healthy people. Moreover, 3D-LVGLS had a stronger correlation with 2D-LVGLS ( $r=0.772, P \otimes 0.0001)$.

\section{Conclusion}

Patients with sepsis or septic shock demonstrated improvement of LV myocardial function during hospitalization, but their myocardial function was not fully recovered to normal on the 10th day. Both 3D and 2D LVGLS could quickly detect changes of patients with covert septic myocardial dysfunction, whereas LVEF could not.

\section{Introduction}


Sepsis is associated with high morbidity and mortality in contemporary ICUs worldwide [1, 2]. Cardiac dysfunction caused by sepsis, usually defined as sepsis cardiomyopathy, is diagnosed in between $40 \%$ and $60 \%$ of septic patients within the first 3 days [3]. A growing body of literature has recognized the danger of cardiomyopathy associated with sepsis and septic shock [4-7]. A large number of studies have described different types of myocardial dysfunction in sepsis. Assessment of the left ventricular function has a pivotal role in treating circulatory impairment, though the prevalence of left ventricular dysfunction varies widely (18-65\%) during sepsis and septic shock [8]. Echocardiography can be used to diagnose and monitor left ventricular dysfunction induced by sepsis [9]. A variety of echocardiographic parameters have been developed to assess left ventricular function [10-12]. LVEF is a common clinical measurement for diagnosing the global systolic function of the left ventricle at present [13]. However, in the early period of sepsis cardiac dysfunction, the ejection fraction is often susceptible to the tiny injury of myocardial movement and is likely to be dependent on the patients' heart rate and various rehydration treatment loads [14].

Strain imaging has become a powerful tool for accurately quantifying cardiac mechanics, as it can provide prognostic information when LVEF is normal or near normal [15]. 2D- STE can evaluate left ventricular function, judge the severity of the disease and determine the value of truncation in order to evaluate the whole approach to disease management. A considerable amount of studies reported that 3D-STE is theoretically superior to 2D-STE for quantifying LV deformation. 3D-STE is fast becoming a key instrument in analyzing full-volume data of the left ventricle [16]. STE imaging used as a research tool to monitor the evolution of myocardial deformation will provide clinicians with a useful additional imaging parameter to facilitate the assessment of patients with covert septic myocardial dysfunction [17].

While LVEF will remain the footstone of LV function assessment, the clinical applicability and reliability of three- and two- dimensional speckle-tracking echocardiography have not been well studied in sepsis or septic shock patients [18]. We further studied the characteristics of myocardial dysfunction in sepsis or septic shock by using echocardiography in intensive care clinical practice. Our approach was to explore the development of myocardial dysfunction by observing the serial changes of three- and twodimensional strain parameters in left ventricular.

\section{Materials And Methods}

\section{Study design and participants}

This prospective observational study was performed at Union Hospital, Tongji Medical College, Huazhong University of Science and Technology, Wuhan, China, between December 2020 and December 2021. 103 Patients ( $\geq 18$ years old) who met sepsis and septic shock criteria defined by the international guidelines for the management of sepsis and septic shock in 2021 were included in the study [19]. Patients with chronic heart failure or severe valvular heart disease were excluded. Chronic heart failure was defined as a baseline (before ICU admission) LVEF below $45 \%$. The study protocols were approved by the institutional Ethical Committee of the Union hospital, Tongji Medical College, Huazhong University of 
Science and Technology, Wuhan, China (2021-S047). All patients provided oral and written informed consent.

\section{Clinical Data}

Demographic data of participants contained age, sex, body mass index (BMI), and body surface area (BSA). Past medical history included hypertension, diabetes mellitus, coronary artery disease, malignancy, chronic liver disease, and chronic kidney disease. The sources of infection included hepato-biliarypancreas, lungs and gastrointestinal tracts. Laboratory findings (Oxygenation index, C reactive protein, arterial blood gas analysis, and lactate concentration, myocardial enzyme et al.) were collected. The severity of illness was assessed according to Acute Physiology and Chronic Health Evaluation (APACHE$\bigotimes$ ) and SOFA scores. Norepinephrine was the first-choice vasopressor therapy (used to target a mean arterial pressure of $65 \mathrm{mmHg}$ or more); dobutamine was added in the presence of decreased LVEF (<45\%) with ongoing signs of hypoperfusion despite adequate mean arterial pressure. All patients were treated according to international guidelines for the treatment of septic shock and after initial resuscitation, at the discretion of the treating clinicians.

\section{Conventional echocardiography}

All echocardiographic examinations included were performed on Philips echocardiographic systems (EPIQ 7C; S5-1, X5-1 transducer; Philips Healthcare, Andover, MA, USA). We separated echocardiographic data based on time intervals, namely, 1, 2, 3, 5, 7 and 10 days after sepsis or septic shock. And the electrocardiogram (ECG) was recorded simultaneously. Two-dimensional LVEF measurements were based on end-systolic/diastolic LV volumes. Transthoracic echocardiographic examinations were performed on commercially available systems.

2D-STE was performed on vendor-independent 2D speckle-tracking software (2D Cardiac Performance Analysis Ver 1.3, TomTec, Germany) based on the previously described method. The longitudinal strain was the average value of three apical projections (four-chamber, two-chamber, and long-axis views). After speckle-tracking analysis of the LV endocardium on a frame-by-frame basis averaged over four cardiac cycles, the software provided regional strain curves, from which the peak regional strain value was calculated. 2D-GLS was calculated as the peak strain value from the averaged strain curves that were generated from 17 segmental strain curves. Adequacy of tracking was verified visually, and if the tracking seemed incorrect, manual adjustment of the endocardial border was performed.

In this study, clear and focused LV apical four chamber images were obtained, and the fan angle was adjusted to ensure they could cover the whole region of interest. The 3D full-volume imaging mode was determined, and the depth and gain of the image were adjusted to keep the image frame frequency $\geq 20 \mathrm{~Hz}$. Four consecutive complete cardiac cycles were collected for 3D image storage. 3D full-volume datasets were analyzed using vendor-independent 3D speckle-tracking software (LV Analysis, 
TOMTEC Imaging Systems GmbH, Unterschleissheim, Germany). In the apical four- and two-chamber views of the end-diastolic frame, the apical point of the LV and the center of the mitral annular line were selected to set the largest LV apical long-axis dimensions and then corresponding landmarks of the aortic annulus were identified in the apical three-chamber view. Subsequently, the software performed 3D speckle-tracking analyses throughout the cardiac cycle. The software provided left ventricular volume and averaged longitudinal strain-time curves.

The parameters used in this study were defined as follows: LVEF was defined as depressed ( $<50 \%$ or when an inotrope infusion was needed to achieve a value $\geq 50 \%$ ), or normal ( $>50 \%$ ). Secondaryhypokinesia was defined as the occurrence of depressed LVEF of one or more hypocardia in 3 days; nonhypokinetics was recorded if LVEF was never depressed [3,20].

\section{Statistical analysis}

Continuous variables were expressed as mean \pm SE or medians, and IQR and categorical variables were expressed as percentages. Shapiro-Wilk test was used to determine whether the data were normally distributed or not. Generalized linear mixed model (GLMM) approach was used to examine the betweengroup differences in the longitudinal trajectories of the echocardiographic measurements over time. Correlations were tested using the Spearman method with Benjamini-Hochberg correction to control the false discovery rate at the 0.05 level. The data were analyzed by IBM SPSS Statistics for Windows (Version 26.0, IBM Corp Armonk, NY, USA).

\section{Results}

\section{Patients Characteristics and clinical data}

Table 1 summarized the main clinical characteristics of patients included. The average age of the 103 patients with sepsis or septic shock was 59 (47 to 68), and 67 (65\%) were men. Past medical history included hypertension, diabetes mellitus, coronary artery disease, malignancy, chronic liver disease, and chronic kidney disease. 23 (22\%) patients had hypertension, 26 (25\%) patients had chronic pulmonary disease, and 31 (30\%) patients had chronic kidney disease. The sources of infection varied, with 34 (33\%) cases of hepato-biliary-pancreas infection, 26 (25\%) pulmonary, 3 (2\%) gastrointestinal, and $12(11 \%)$ unknown. During the period of medical treatment, 50 (49\%) patients were given norepinephrine, 18 (17\%) patients dobutamine, 8 (7\%) patients levosimendan, and 4 (3\%) patients amiodarone. All patients' SOFA improved within 10 days as shown in Figure 1. Most patients had organ function damage, with laboratory data showing changes in abnormalities within 10 days, including lactate, D-dimer, C-reactive protein and procalcitonin, urea nitrogen, creatinine, glomerular filtration rate, and cardiac enzymes scan results (Table 2). 


\section{Serial Changes in LV Volumes and EF Analysis by 2D and 3D}

A total of 88 adult patients for 2D-STE and 3D-STE echocardiography were divided into two groups according to their wall motion status: the non-hypokinetics group and the secondary hypokinesia group (Table 3). Conventional echocardiograms were analyzed during hospitalization, on the 1st day (nonhypokinetics, $n=41$; secondary hypokinesia, $n=47$ ), the 3rd day (non-hypokinetics, $n=38$; secondary hypokinesia, $n=42$ ), the 5 th day (non-hypokinetics, $n=33$; secondary hypokinesia, $n=30$ ), the 7th-10th day (non-hypokinetics, $\mathrm{n}=32$ secondary hypokinesia, $\mathrm{n}=27$ ).

All measurements of LV function were abnormal immediately after sepsis or septic shock and improved over the 10 days. 2D-LVEF demonstrated a progressive, stepwise improvement each time point assessed $\left(P_{\text {time }}=0.033, P_{\text {group }}=0.041\right.$, respectively $)$. Although $3 \mathrm{D}$ slightly underestimated the $\mathrm{LV}$ volumes at each time point, it displayed the same changing tendency during the 10 days (3D-LVEF, $P_{\text {time }}=0.002, P_{\text {group }}=0.011$, respectively). As depicted in Figure 2, both $2 \mathrm{D}$ and 3D analyses showed that, compared to the 1 st day, all patients had stepwise improvement at last time point assessed.

\section{Left Ventricular Systolic Myocardial Deformation by 3D and 2D strain}

Both 3D and 2D Strain analyses of left ventricular systolic myocardial deformation showed a significant improvement within the 10 days (Table 3): 2D-LVGLS respectively, $P_{\text {time }}=0.000, P_{\text {group }}=0.018,2 \mathrm{D}$-LVGCS respectively, $P_{\text {time }}=0.000, P_{\text {group }}=0.332 ; 3 \mathrm{D}$-LVGLS respectively, $P_{\text {time }}=0.000, P_{\text {group }}=0.391$, 3D-LVGCS respectively, $P_{\text {time }}=0.022, P_{\text {group }}=0.3362$. Figure 3 showed the comparison between non-hypokinetics group and secondary hypokinesia group using 3D and 2D GLS on the 1st Day. Both 3D and 2D LVGLS could quickly detect changes of patients with covert septic myocardial dysfunction, whereas LVEF could not.

\section{Comparison between the 2 groups by 3D and 2D Strain}

The level of left ventricular systolic myocardial deformation of both groups first decreased and then increased during hospitalization, but both 3D and 2D strains imaged at the last time point assessed were lower than normal (Figure 4). Compared to the 1st day, 2D strains of non-hypokinetics were: GLS $\left[P_{3 \mathrm{th}}=0.000, P_{5 \mathrm{th}}=0.029, P_{7-10 \mathrm{th}}=0.001\right]$, GCS $\left[P_{3 \mathrm{th}}=0.007, P_{5 \mathrm{th}}=0.046, P_{7-10 \mathrm{th}}=0.881\right] ; 2 \mathrm{D}$ strains of secondary hypokinesia GLS $\left[P_{3 \mathrm{th}}=0.000, P_{5 \mathrm{th}}=0.233, P_{7-10 \mathrm{th}}=0.419\right]$, GCS $\left[P_{3 \mathrm{th}}=0.184, P_{5 \mathrm{th}}=0.633, P_{7-}\right.$ 10th $=0.122$. We found 3D strains displayed a same changing tendency: compared to 1 st day, 3D strains of non-hypokinetics were: GLS $\left[P_{3 \text { th }}=0.128, P_{5 \text { th }}=0.962, P_{7-10 \mathrm{th}}=0.000\right]$, GCS $\left[P_{3 \mathrm{th}}=0.405, P_{5 \text { th }}=0.843, P_{7-}\right.$ 
10 th $=0.687]$; 3D strains of secondary hypokinesia were: GLS $\left[P_{3 \text { th }}=0.662, P_{5 \text { th }}=0.005, P_{7-10 \text { th }}=0.000\right]$, GCS [ $\left.P_{3 \text { th }}=0.033, P_{5 \text { th }}=0.409, P_{7-10 \text { th }}=0.801\right]$.

\section{Applicability and Feasibility of 3D-STE}

3D-STE-derived LVGLS had a stronger correlation with 2D-STE-derived LVGLS ( $r=0.772, P \otimes 0.0001)$, and LVGCS ( $r=0.289, P \otimes 0.0001)$. Besides, 3D-LVGLS had smaller biases and narrower LOAs values in comparison to 2D-LVGLS (bias: -2.946 and 2.482, LOA: -5.551 and 5.087, respectively). Comparison between 3D-LVGCS and 2D-LVGLS values (bias: -8.773 and 3.791, LOA: -14.80 and 9.822, respectively) was shown in Figure 5.

\section{Discussion}

To the best of our knowledge, this is the first comprehensive continuous observational study using 3D and 2D strain for left ventricular myocardial deformation assessment in sepsis and septic shock populations. The main findings of this study are as follows: (1) for sepsis and septic shock, both 3D and 2D-LV GLS could quickly detect changes in patients with covert septic myocardial dysfunction, whereas LVEF could not; (2) there was gradual improvement of LV myocardial function during hospitalization, but it was not fully recovered to normal on the 10th day.

Diagnosis of sepsis in the ICU can be challenging due to pre-existing organ dysfunction, pre-admission treatment, and concurrent organ support [21]. First, in our cohort past medical history included hypertension, diabetes mellitus, coronary artery disease, malignancy, chronic pulmonary disease chronic kidney disease. Then, there were various infection sources, including 34 (33\%) cases of hepato-biliarypancreas infection, 26(25\%) pulmonary, and $12(11 \%)$ unknow. Finally, during the period of medical treatment, patients were prescribed different medications: 50 (49\%) patients were given norepinephrine, 18 (17\%) dobutamine, 8 (7\%) levosimendan, and 4 (3\%) amiodarone, which implies a possibility influence of medication on changes in laboratory data and abnormalities during the 10 days. A possible explanation for this might be the complex interplay between host, pathogen, and therapeutic interventions -their ultimate effects may be the results of different mechanisms [22]. We also found that SOFA got worse before it eventually turned for the better. The role of baselines in patients with sepsis or septic shock also needs to be scrutinized.

\section{The role of GLS in sepsis or septic shock}

One of our findings was that both 3D and 2D-LV GLS could quickly detect changes of patients with covert septic myocardial dysfunction, whereas LVEF could not. Since the first clinical demonstrations of septic myocardial depression in the 1980s [23], the role of left ventricular dysfunction in poor prognosis remains controversial. Left ventricular systolic and diastolic dysfunction have been extensively studied previously in patients with sepsis and septic shock. Vieillard-Baron found that LVEF was not associated with 
prognosis in left ventricular dysfunction [24]. In contrast, Furian demonstrated a correlation between poor prognosis and left ventricular dysfunction [25]. While the study of Ronaldo in 2014 showed that LVEF in left ventricular systolic dysfunction related to sepsis patients was neither a sensitive indicator nor an independent predictor of death, many researchers believe the conclusion had limitations and may be the result of the small sample size and the specific situations of the study [26]. Preliminary data of left ventricular diastolic dysfunction assessed by tissue Doppler imaging has been confirmed to be associated with mortality; however, the evidence at the point is limited [27]. Among various parameters, ejection fraction is most commonly used to assess left ventricular systolic function, but controversies exist [28].

Septic cardiomyopathy on clinical applications in the adult ICU has not been expounded clearly, and the real nature of this special septic organ failure remains unclear [29]. However, GLS has been found as a reliable echocardiographic index for evaluating left ventricular function and the whole approach to disease management in patients with sepsis or septic shock. Kalam demonstrated that assessing GLS from speckle tracking analysis of two-dimensional echocardiography has become a clinically viable alternative to LVEF for measuring myocardial function. GLS is more sensitive to left ventricular dysfunction than LVEF and has a certain prognostic value [30]. Hai demonstrated that Speckle tracking echocardiography could detect early subclinical left ventricular systolic dysfunction in patients with septic shock by overall left ventricular longitudinal strain, superior to conventional echocardiographic parameters [31]. Lina demonstrated that GLS peaks were frequently reduced in patients with septic shock, alone or in combination with LVEF. It is associated with LVEF and NT-proBNP. GLS provide further insights into the characteristics of myocardial dysfunction in septic shock [32]. The advantage of GLS is that it provides more details on myocardial movement, and GLS has been proposed as a trial of choice in guidelines for monitoring asymptomatic cardiotoxicity.

Consistent with the researches of the above-mentioned scholars, our study focused on a series of consecutive cases with two- and three-dimensional echocardiography, and suggested that compared to LVEF, GLS was more sensitive in detecting covert septic myocardial dysfunction.

\section{Serial changes in left ventricular myocardial}

In 2016, the guidelines issued by the SCCM and ES-ICM together with more than 10 professional critical care organizations around the world clearly proposed that early intervention for sepsis cardiac dysfunction is the key to treatment [9]. Our second major finding was that patients demonstrated gradual improvement of LV myocardial function during hospitalization, but their myocardial function was not fully recovered to normal on the 10th day. As suggested by Landesberg G, myocardial injury is manifestation of cardiovascular dysfunction in sepsis [33]. The results of this research support the idea that myocardial dysfunction during septic shock could be described as a state of left ventricular depression, revealing reversibility on remissions [34]. Improvement in the diagnosis of left ventricular 


\section{Applicability and feasibility of 3D-STE in sepsis and septic shock}

Since the publication of the Consensus of the European Association of Cardiovascular Imaging (EACVI)/ American Society of Echocardiography (ASE), strain imaging has become a powerful tool for accurately quantifying cardiac mechanics. 3D echocardiographic measurements are now recommended for patients with good image quality [15]. With respect to the first research question, it was found that 3D-STE-derived LVGLS had a stronger correlation with 2D-STE-derived LVGLS. Besides, 3D-LVGLS had smaller biases and narrower LOAs values in comparison to 2D-LVGLS. The relevance of 3D-GLS is clearly supported by the current findings.

Non-invasive evaluation of ventricular function is still the core of modern cardiology, and there are good reasons to include GLS in clinical decision making [35]. For patients with sepsis cardiac dysfunction, future studies could rely more on GLS to evaluate left ventricular function, judge the severity of the disease and determine the value of truncation in order to evaluate the whole approach to disease management. Nevertheless, this study had some limitations. It was a single-center study, and the sample size was relatively small. Our study was limited to exploring the normal 3D-STE mechanical functions in sepsis and septic shock patients during 10 days. Furthermore, 3D-STE is dependent on image quality. Moreover, the comparability of STE among different vendors is uncertain. The lack of clear and unified diagnostic criteria for left ventricular myocardial deformation in sepsis and septic shock is the fundamental reason that restricts the research in this field. Certain issues need to be considered for the diagnostic value of speck tracking in septic cardiac dysfunction. Although 3D-STE has a considerable prospect, there is still a lack of consistency [36]. How to effectively evaluate the cardiac function of sepsis patients is related to significant differences in statistical prognostic outcome indicators, study specificity, observation methodology, and detection time points.

\section{Conclusion}

Patients with sepsis or septic shock demonstrated improvement of LV myocardial function during hospitalization, but their myocardial function was not fully recovered to normal on the 10th day. Both 3D and 2D LVGLS could quickly detect changes of patients with covert septic myocardial dysfunction, whereas LVEF could not.

\section{Abbreviations}

ICU: intensive Care Unit;

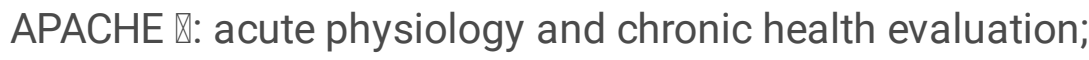


SOFA: sequential organ failure assessment;

EDVI: end-diastolic volume index;

ESVI: end-systolic volume index;

EF: ejection fraction;

2D: two-dimensional;3D: Three-dimensional;

STE: speckle tracking echocardiography;

2D-STE: 2-dimensional speckle-tracking echocardiography;

3D-STE: 3-dimensional speckle-tracking echocardiography;

GLS: global longitudinal strain;

GCS: global circumferential strain;

\section{Declarations}

\section{Ethical Approval and Consent to participate}

The study protocols were approved by the institutional Ethical Committee of the Union hospital, Tongji Medical College, Huazhong University of Science and Technology, Wuhan, China (2021-S047). All patients provided oral and written informed consent.

\section{Consent for publication}

All authors approved it for publication.

\section{Availability of supporting data}

The data that support the findings of this study are available from Mingxing Xie, MD, PhD, FASE, FAHA, FACC. but restrictions apply to the availability of these data, which were used under license for the current study, and so are not publicly available. Data are however available from the authors upon reasonable request and with permission of Mingxing Xie Mingxing Xie,MD, PhD, FASE, FAHA, FACC.

\section{Competing interests}

None declared. 


\section{Funding}

This work was supported by the National Natural Science Foundation of China (Grant Nos. 81727805, 81922033), the Shenzhen Science and Technology under Grant (SGDX20190917094601717 and JCYJ20210324141216040).

\section{Authors' contributions}

All authors listed have made a substantial, direct and intellectual contribution to the work. Xiaojun Yan,Yuman Li,Juanjuan Liu and Ting Zhou contributed equally to this manuscript. Yi Zhou,Wei Sun,Chenchen Sun,and Jing Ma prepared tables1-3 and figures1-5. Li Zhang,You Shang and Mingxing Xie critically reviewed the manuscript for important intellectual content.All authors listed have made a substantial, direct and intellectual contribution to the work, and approved it for publication.

\section{Acknowledgements}

Not applicable.

\section{References}

1. Singer M, Deutschman CS, Seymour CW, Shankar-Hari M, Annane D, Bauer M, et al. The Third International Consensus Definitions for Sepsis and Septic Shock (Sepsis-3). JAMA. 2016;315:801-10.

2. Rudd KE, Johnson SC, Agesa KM, Shackelford KA, Tsoi D, Kievlan DR, et al. Global, regional, and national sepsis incidence and mortality, 1990-2017: analysis for the Global Burden of Disease Study. The Lancet. 2020;395:200-11.

3. Vieillard-Baron A, Caille V, Charron C, Belliard G, Page B, Jardin F. Actual incidence of global left ventricular hypokinesia in adult septic shock. Crit Care Med. 2008;36:1701-6.

4. Cuthbertson Bh Fau - Elders A, Elders A Fau - Hall S, Hall S Fau - Taylor J, Taylor J Fau - MacLennan G, MacLennan G Fau - Mackirdy F, Mackirdy F Fau - Mackenzie SJ, et al. Mortality and quality of life in the five years after severe sepsis. Crit Care.2013;17: R70.

5. Beesley SJ, Weber G, Sarge T, Nikravan S, Grissom CK, Lanspa MJ, et al. Septic Cardiomyopathy. Crit Care Med. 2018;46:625-34.

6. Vallabhajosyula S, Gillespie SM, Barbara DW, Anavekar NS, Pulido JN. Impact of New-Onset Left Ventricular Dysfunction on Outcomes in Mechanically Ventilated Patients With Severe Sepsis and Septic Shock. J Intensive Care Med.2018;33:680-686.

7. Vallabhajosyula S, Shankar A, Vojjini R, Cheungpasitporn W, Sundaragiri PR, DuBrock HM, et al. Impact of Right Ventricular Dysfunction on Short-term and Long-term Mortality in Sepsis: A Metaanalysis of 1,373 Patients. Chest. 2021;159:2254-63. 
8. Aneman A, Vieillard-Baron A. Cardiac dysfunction in sepsis. Intensive Care Med. 2016;42:2073-6.

9. Rhodes A, Evans LE, Alhazzani W, Levy MM, Antonelli M, Ferrer R, et al. Surviving Sepsis Campaign: International Guidelines for Management of Sepsis and Septic Shock: 2016. Intensive Care Med. 2017;43:304-77.

10. Vignon P, Huang SJ. Global longitudinal strain in septic cardiomyopathy: the hidden part of the iceberg? Intensive Care Med. 2015;41:1851-3.

11. Orde Sr Fau - Pulido JN, Pulido Jn Fau - Masaki M, Masaki M Fau - Gillespie S, Gillespie S Fau Spoon JN, Spoon Jn Fau - Kane GC, Kane Gc Fau - Oh JK, et al. Outcome prediction in sepsis: speckle tracking echocardiography based assessment of myocardial function. Critical Care 2014;18: R149

12. de Braga Lima Carvalho Canesso M, Borges IN, de Deus Queiroz Santos TA, Ris TH, de Barros MVL, Nobre $\mathrm{V}$, et al. Value of speckle-tracking echocardiography changes in monitoring myocardial dysfunction during treatment of sepsis: potential prognostic implications. Int J Cardiovasc Imaging.2019;35:855-859.

13. Hollenberg SM, Singer M. Pathophysiology of sepsis-induced cardiomyopathy. Nat Rev Cardiol. 2021;18:424-34.

14. Eguia E, Bunn C, Kulshrestha S, Markossian T, Durazo-Arvizu R, Baker MS, et al. Trends, Cost, and Mortality From Sepsis After Trauma in the United States: An Evaluation of the National Inpatient Sample of Hospitalizations, 2012-2016. Crit Care Med. 2020;48:1296-303.

15. Lang RM, Badano LP, Mor-Avi V, Afilalo J, Armstrong A, Ernande L, et al. Recommendations for cardiac chamber quantification by echocardiography in adults: an update from the American Society of Echocardiography and the European Association of Cardiovascular Imaging. J Am Soc Echocardiogr. 2015;28:1-39 e14.

16. Otto CM, Nishimura RA, Bonow RO, Carabello BA, Erwin JP, Gentile F, et al. 2020 ACC/AHA Guideline for the Management of Patients With ValvularHeart Disease. Journal of the American College of Cardiology. 2021;77:e25-e197.

17. Ehrman RR, Moore SC, Favot MJ, Akers KG, Gallien JZ, Welch RD, et al. Scientific Letter to the Editor: Need for a Definitive Study of Global Longitudinal Strain for Prognostication in Septic Cardiomyopathy. J Am Soc Echocardiogr. 2019;32:549-52 e3.

18. Vallabhajosyula SA-O, Rayes HA, Sakhuja A, Murad MH, Geske JB, Jentzer JC. Global Longitudinal Strain Using Speckle-Tracking Echocardiography as a Mortality Predictor in Sepsis: A Systematic Review. J Intensive Care Med.2019;34:87-93.

19. Evans L, Rhodes A, Alhazzani W, Antonelli M, Coopersmith CM, French C, et al. Surviving sepsis campaign: international guidelines for management of sepsis and septic shock 2021. Intensive Care Med. 2021;47:1181-247.

20. Boissier F, Razazi K, Seemann A, Bedet A, Thille AW, de Prost N, et al. Left ventricular systolic dysfunction during septic shock: the role of loading conditions. Intensive Care Med. 2017;43:633-42.

21. Seymour CW, Liu VX, Iwashyna TJ, Brunkhorst FM, Rea TD, Scherag A, et al. Assessment of Clinical Criteria for Sepsis: For the Third International Consensus Definitions for Sepsis and Septic Shock 
(Sepsis-3). JAMA. 2016;315:762-74.

22. Wilhelm J, Hettwer S, Schuermann M, Bagger S, Gerhardt F, Mundt S, et al. Severity of cardiac impairment in the early stage of community-acquired sepsis determines worse prognosis. Clin Res Cardiol. 2013;102:735-44.

23. Parker Mm Fau - Shelhamer JH, Shelhamer Jh Fau - Bacharach SL, Bacharach SI Fau - Green MV, Green Mv Fau - Natanson C, Natanson C Fau - Frederick TM, Frederick Tm Fau - Damske BA, et al. Profound but reversible myocardial depression in patients with septic shock. Ann Intern Med. 1984; 100:483-90.

24. Vieillard-Baron A, Caille V Fau - Charron C, Charron C Fau - Belliard G, Belliard G Fau - Page B, Page B Fau - Jardin F, Jardin F. Actual incidence of global left ventricular hypokinesia in adult septic shock. Crit Care Med. 2008; 36:1701-6.

25. Furian T, Aguiar C Fau - Prado K, Prado K Fau - Ribeiro RVP, Ribeiro Rv Fau - Becker L, Becker L Fau Martinelli N, Martinelli N Fau - Clausell N, et al. Ventricular dysfunction and dilation in severe sepsis and septic shock: relation to endothelial function and mortality. J Crit Care. 2012;27:319.e9-15.

26. Sevilla Berrios RA, O'Horo JC, Velagapudi V, Pulido JN. Correlation of left ventricular systolic dysfunction determined by low ejection fraction and 30-day mortality in patients with severe sepsis and septic shock: a systematic review and meta-analysis. J Crit Care. 2014;29:495-9.

27. Sturgess DJ, Marwick Th Fau - Joyce C, Joyce C Fau - Jenkins C, Jenkins C Fau - Jones M, Jones M Fau - Masci P, Masci P Fau - Stewart D, et al. Prediction of hospital outcome in septic shock: a prospective comparison of tissue Doppler and cardiac biomarkers.Crit Care. 2010;14:R44.

28. Clancy DJ, Scully T, Slama M, Huang S, McLean AS, Orde SR. Application of updated guidelines on diastolic dysfunction in patients with severe sepsis and septic shock. Ann Intensive Care.2017;7:121.

29. Lanspa MJ, Olsen TD, Wilson EL, Leguyader ML, Hirshberg EL, Anderson JL, et al. A simplified definition of diastolic function in sepsis, compared against standard definitions. J Intensive Care. 2019;7:14.

30. Kalam K, Otahal P, Marwick TH. Prognostic implications of global LV dysfunction: a systematic review and meta-analysis of global longitudinal strain and ejection fraction. Heart. 2014;100:167380.

31. Hai PA-OX, Phuong LL, Dung NM, Hoa LTV, Quyen DV, Chinh NX, et al. Subclinical Left Ventricular Systolic Dysfunction in Patients with Septic Shock Based on Sepsis-3 Definition: A Speckle-Tracking Echocardiography Study.Crit Care Res Pract.2020; 2020:6098654.

32. De Geer L, Engvall J, Oscarsson A. Strain echocardiography in septic shock - a comparison with systolic and diastolic function parameters, cardiac biomarkers and outcome. Crit Care. 2015;19:122.

33. Landesberg G, Levin PD, Gilon D, Goodman S, Georgieva M, Weissman C, et al. Myocardial Dysfunction in Severe Sepsis and Septic Shock: No Correlation With Inflammatory Cytokines in Reallife Clinical Setting. Chest. 2015;148:93-102.

34. Parrillo Je Fau - Burch C, Burch C Fau - Shelhamer JH, Shelhamer Jh Fau - Parker MM, Parker Mm Fau - Natanson C, Natanson C Fau - Schuette W, Schuette W. A circulating myocardial depressant 
substance in humans with septic shock. Septic shock patients with a reduced ejection fraction have a circulating factor that depresses in vitro myocardial cell performance. J Clin Invest.1985; 76:153953.

35. Cecconi M, Evans L, Levy M, Rhodes A. Sepsis and septic shock. The Lancet. 2018;392:75-87.

36. Balasubramanian S, Punn R, Smith SN, Houle H, Tacy TA. Left Ventricular Systolic Myocardial Deformation: A Comparison of Two- and Three-Dimensional Echocardiography in Children. J Am Soc Echocardiogr. 2017;30:974-83.

\section{Figures}




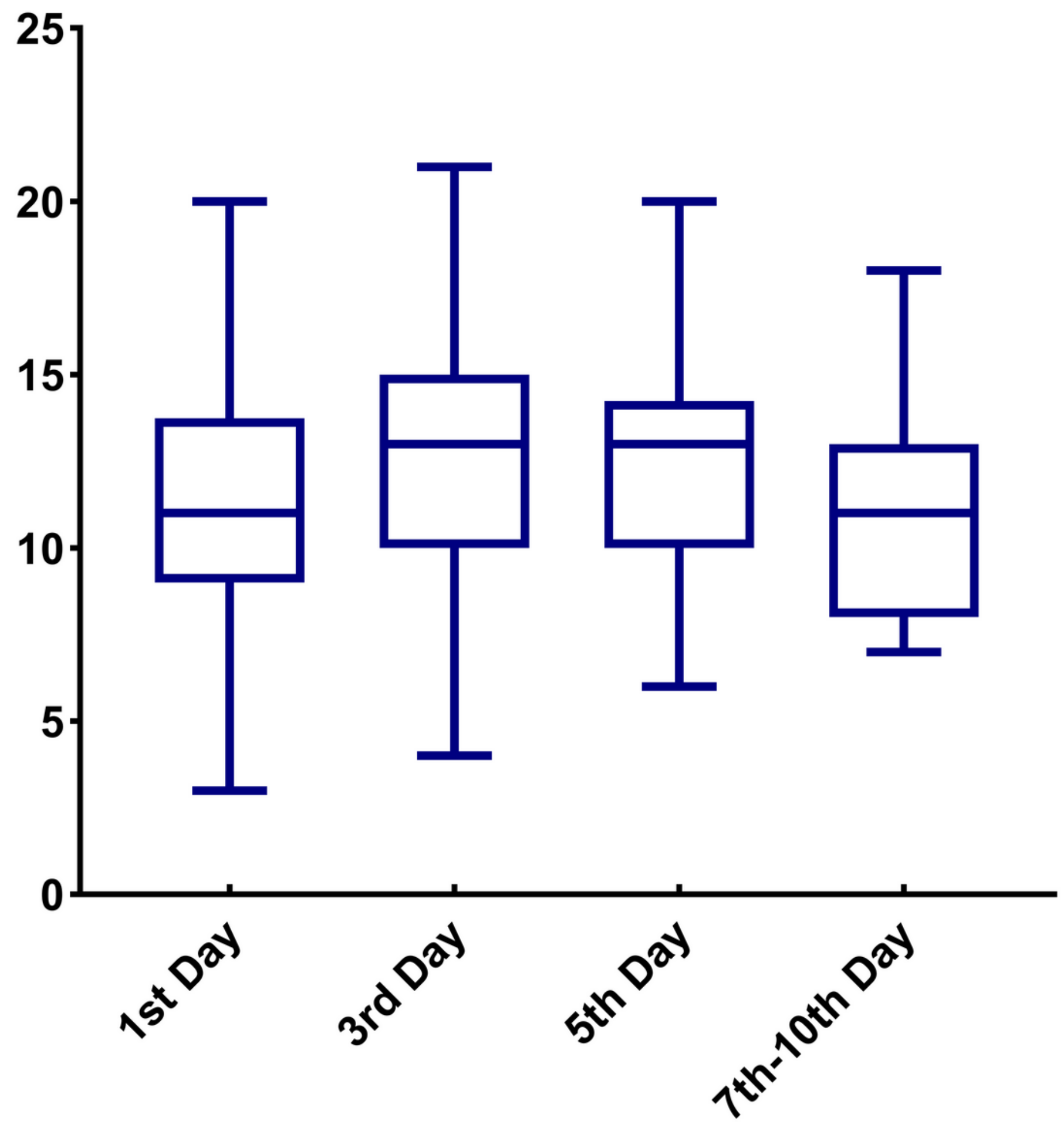

Figure 1

Sequential organ failure assessment (SOFA) change over time in patients studied.

Box and whisker plots of the minimum, 25th percentile, median, 75th percentile, maximum and change over time of sequential organ failure assessment (SOFA) at each time point after sepsis and septic shock. 
A

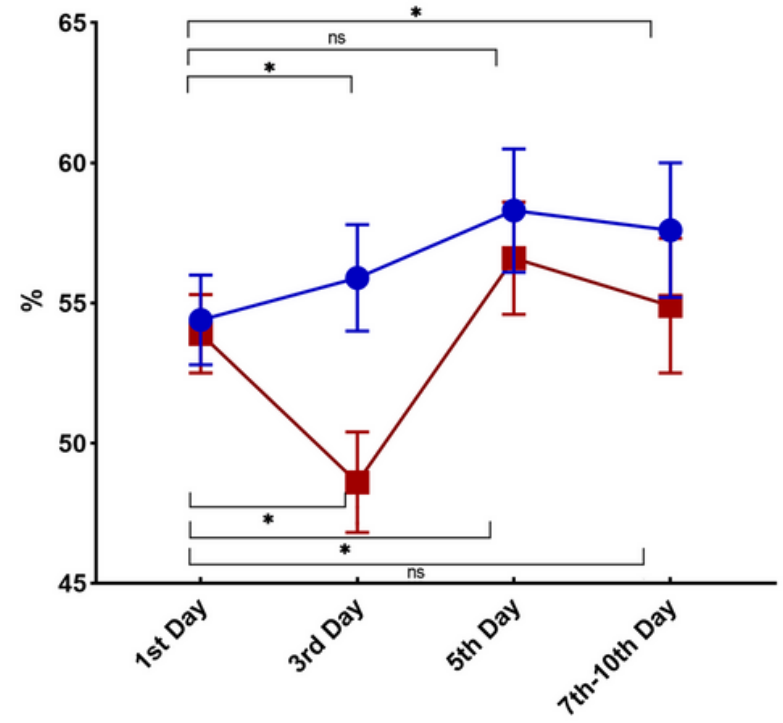

2D-LVEF(\%)
B

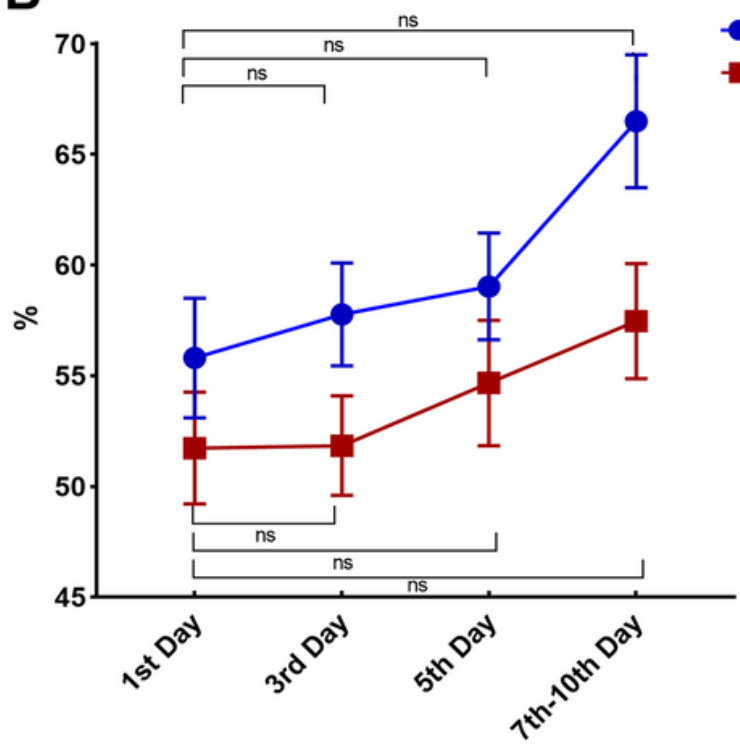

3D-LVEF(\%)

Figure 2

\section{D-LVEF and 2D-LVEF serial changes over time.}

Margin plots with $95 \%$ confidence interval in reference to time after sepsis or septic shock in nonhypokinetics group and secondary hypokinesia group. (A) 2D-left ventricular function, (B) 3D- left ventricular function. The index was considered statistical significance at *p $₫ 0.05$ compared to the 1 st day, each group respectively. 


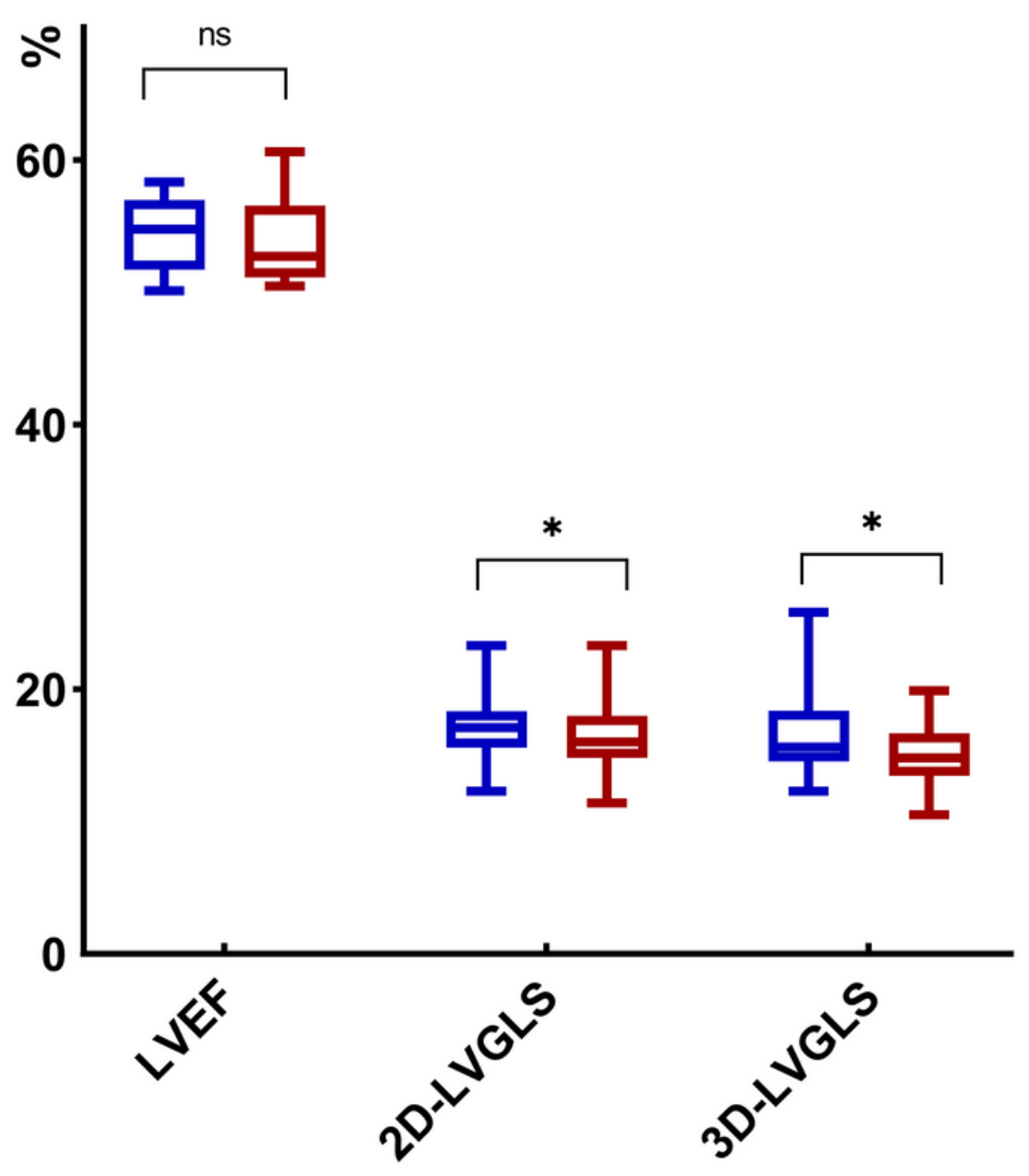

№n-hypokinetics

드 Secondary hypokinesia

\section{Figure 3}

Comparison between Non-hypokinetics group and Secondary hypokinesia group by 3D and 2D GLS on the 1st Day

Left ventricle ejection fraction (EF) and absolute values of left ventricle global longitudinal strain at the 1 st day of sepsis and septic shock. Box and whisker plots of the minimum, 25th percentile, median, 75th percentile, maximum, generalized linear mixed model (GLMM) modeling approach was used to examine the between-group, $P<0.05$ was considered to indicate statistical significance. 

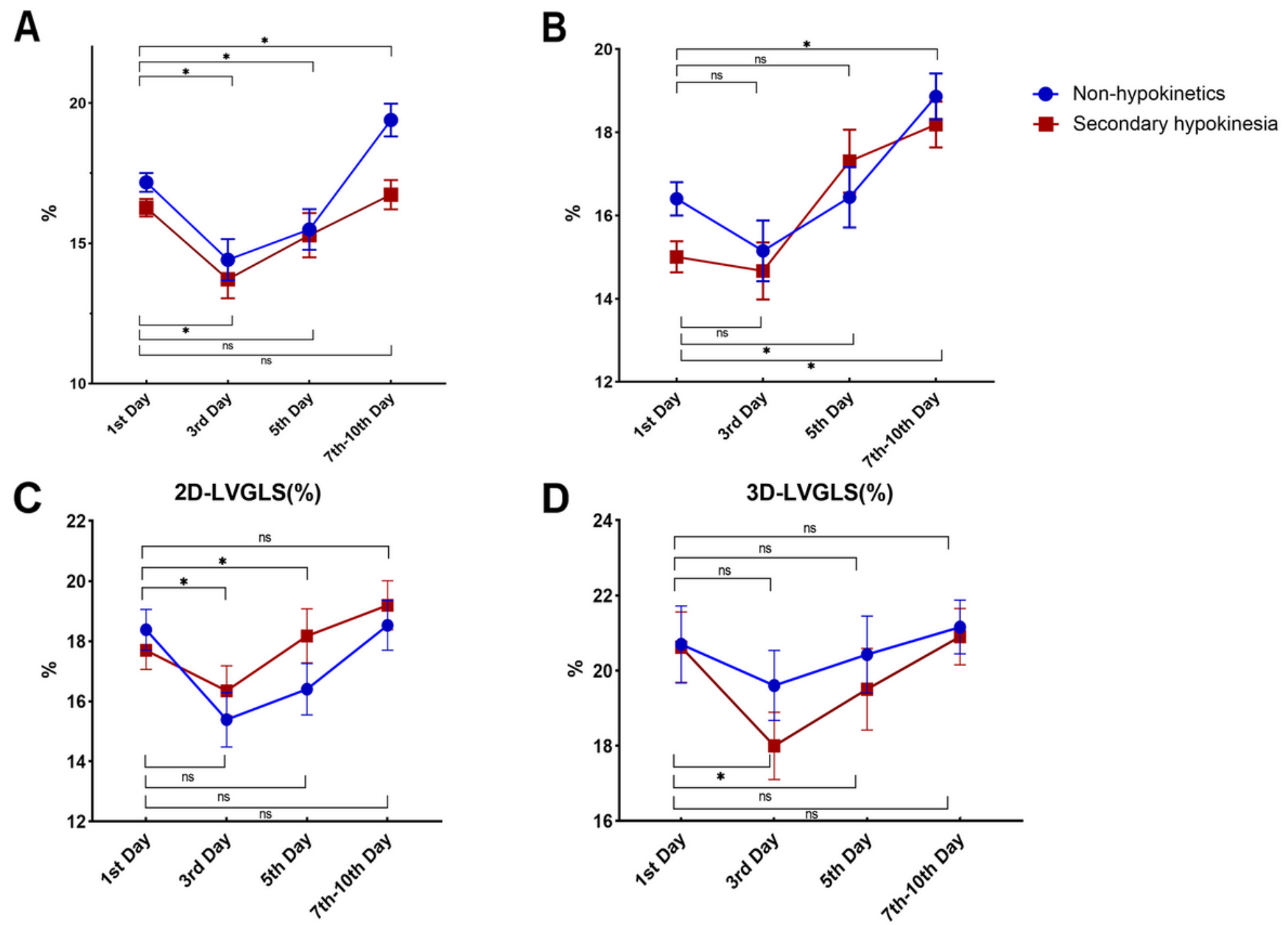

2D-LVGCS(\%)

3D-LVGCS(\%)

\section{Figure 4}

\section{D-STE and 2D-STE serial changes over time.}

Margin plots with 95\% confidence interval in reference to time after sepsis or septic shock in nonhypokinetics group and secondary hypokinesia group. (A) 2D-LVGLS, Two-dimensional left ventricular function global longitudinal strain (B) 3D-LVGLS, Three-dimensional left ventricular function global longitudinal strain. (C) 2D-LVGCS, Two-dimensional left ventricular function global circumferential strain. (D) 3D-LVGCS, Three-dimensional left ventricular function global circumferential strain. The index was considered statistical significance at * $\mathrm{p} \otimes 0.05$ compared to the 1 st day, each group respectively. 
A

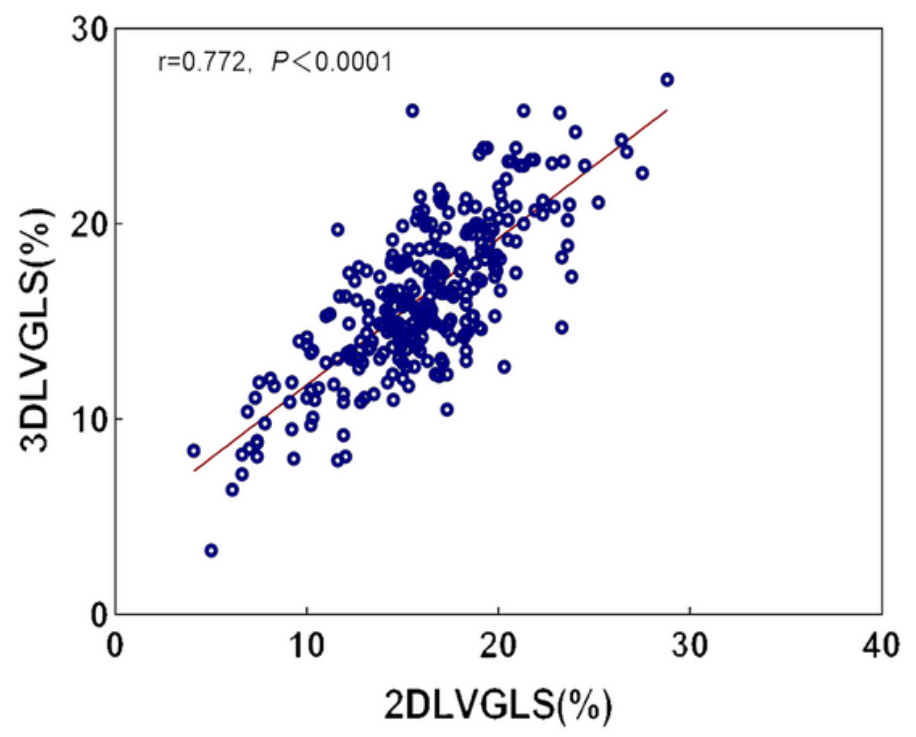

C

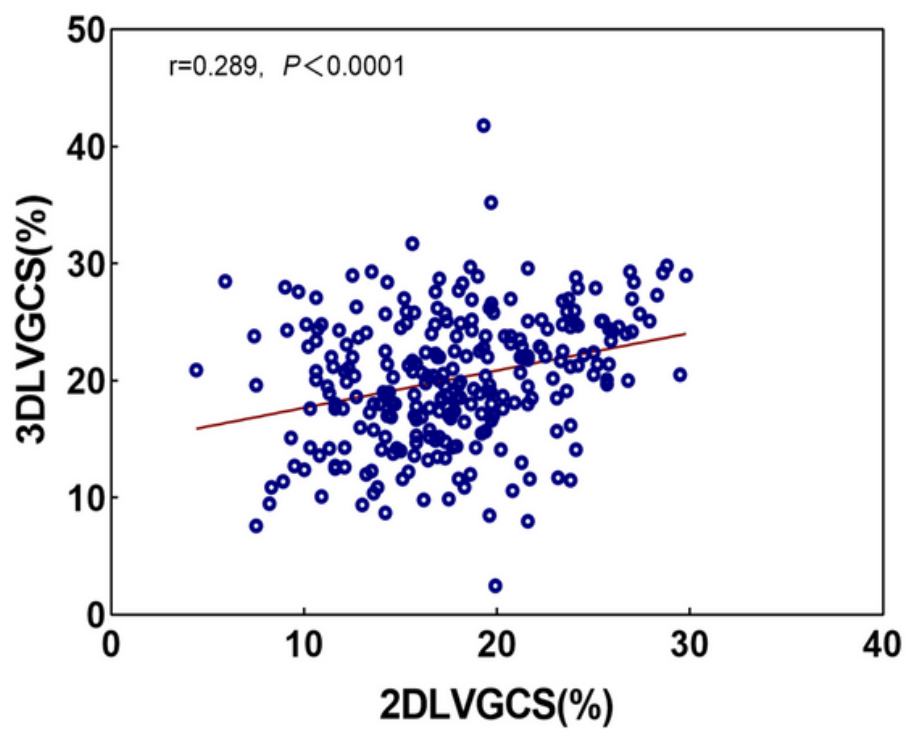

B

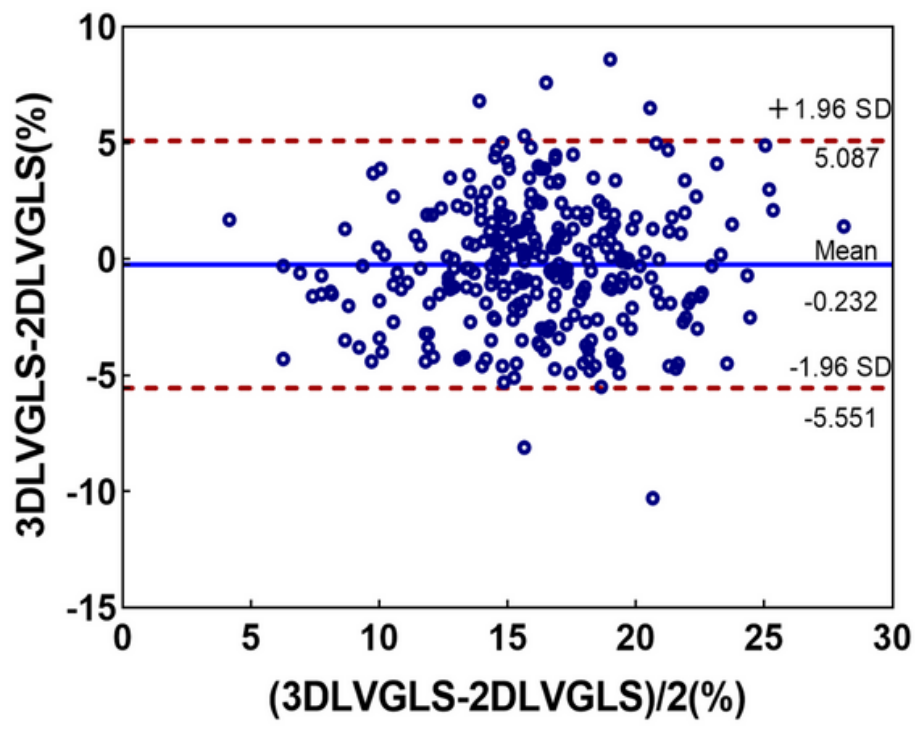

D

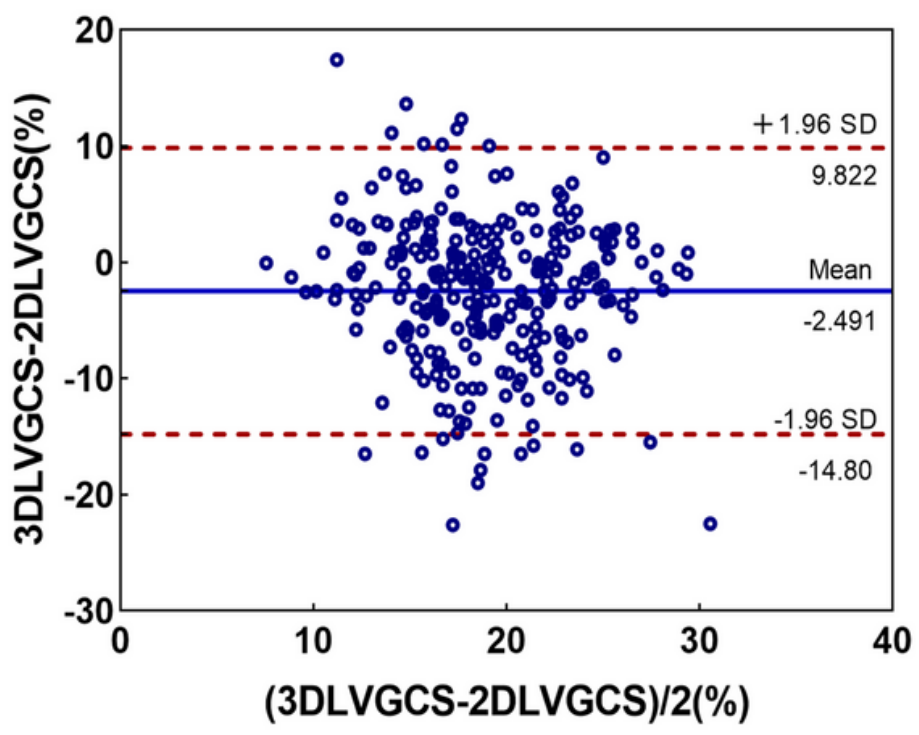

Figure 5

Correlation and Bland-Altman analysis for 3D-STE and 2D-STE

(A) The correlation between 2D-LVGLS and 3D-LVGLS;(B) The consistence between 2D-LVGLS and 3DLVGLS;(C) The correlation between 2D-LVGCS and 3D-LVGCS;(D) The consistence between 2D-LVGCS and 3D-LVGCS. 